\title{
Region-Based Iterative Reconstruction of Structurally Changing Objects in CT
}

\author{
Geert Van Eyndhoven, Kees Joost Batenburg, and Jan Sijbers
}

\begin{abstract}
X-ray computed tomography (CT) is a powerful tool for noninvasive imaging of time-varying objects. In the past, methods have been proposed to reconstruct images from continuously changing objects. For discretely or structurally changing objects, however, such methods fail to reconstruct high quality images, mainly because assumptions about continuity are no longer valid. In this paper, we propose a method to reconstruct structurally changing objects. Starting from the observation that there exist regions within the scanned object that remain unchanged over time, we introduce an iterative optimization routine that can automatically determine these regions and incorporate this knowledge in an algebraic reconstruction method. The proposed algorithm was validated on simulation data and experimental $\mu \mathrm{CT}$ data, illustrating its capability to reconstruct structurally changing objects more accurately in comparison to current techniques.
\end{abstract}

Index Terms-Computed tomography (CT), tomography, iterative reconstruction, region estimation.

\section{INTRODUCTION}

$\mathbf{I}$ $\mathrm{N}$ COMPUTED tomography (CT), most reconstruction techniques assume that the object does not deform during the acquisition of projection data. If the scanned object is subject to deformation or structural changes, however, these techniques are no longer adequate and the reconstructed image will suffer from artefacts such as blurring.

Current approaches to account for deformation of the object can be classified into two categories. In a first class of methods, which we refer to as the deformation-based techniques, a deformation model is incorporated in the reconstruction process. Affine transformations can be modeled directly by adjusting the projection data [1] and subsequently using a standard reconstruction algorithm. Many a priori known invert-

Manuscript received March 22, 2013; revised July 23, 2013 and October 4, 2013; accepted December 17, 2013. Date of publication January 2, 2014; date of current version January 13, 2014. The work of K. J. Batenburg was supported by the Netherlands Fund for Scientific Research (NWO), project number 639.072.005. The work of J. Sijbers was supported by the IWT SBO TomFood project. The associate editor coordinating the review of this manuscript and approving it for publication was Prof. Edwin Marengo. (Corresponding author: G. Van Eyndhoven.)

G. Van Eyndhoven and J. Sijbers are with the iMinds-Vision Lab, University of Antwerp, B-2610 Wilrijk, Belgium (email: geert.vaneyndhoven@uantwerpen.be; jan.sijbers@uantwerpen.be).

K. J. Batenburg is with Centrum Wiskunde \& Informatica, NL-1090 GB Amsterdam, The Netherlands. He is also with the iMinds-Vision Lab, University of Antwerp, B-2610 Wilrijk, Belgium and Mathematical Institute, Leiden University, NL-2300 RA Leiden, the Netherlands (e-mail: joost.batenburg@cwi.nl).

Color versions of one or more of the figures in this paper are available online at http://ieeexplore.ieee.org.

Digital Object Identifier 10.1109/TIP.2013.2297024 ible deformation models can be compensated for by modifying classical algorithms like Filtered BackProjection (FBP) or Simultaneous Iterative Reconstruction Technique (SIRT) [2], [3]. If no a priori deformation model is available, the deformation parameters can be estimated using a series of different techniques [1], [4]-[8]. A second class of methods generates reconstructions using different subsets of all available projection data, assuming that each of these subsets contains data acquired from a motionless object. We refer to this type of methods as subset-reconstruction techniques. In medical imaging, this technique is usually known as phase binning [9], where the subsets are obtained by ordering the projection data per phase, assuming a periodic motion. Alternatively, different subsequent scans can be performed in order to obtain the subsets, assuming the object remained unchanged during each scan. Examples of this technique can be found in non-destructive material tests [10], soil structure and water retention studies [11] or observations of root growth [12]. Reconstruction quality of standard subset-reconstruction techniques can be improved by enforcing similarity among the subsets with regularization strategies [13]. Another improvement can be made if a prior, high quality reconstruction is available, e.g. based on projection data acquired from the object before the changes have commenced. From this prior reconstruction, projections can be simulated and subtracted from the projection data acquired from the time-varying object. The changing volume can then be reconstructed by applying a sparse reconstruction technique to the resulting projection difference [14].

The deformation-based technique is only applicable to problems where continuous deformations deteriorate reconstruction quality, whereas the subset-reconstruction technique can only be applied to periodic motion or under the assumption that the object remains unchanged during the acquisition of projection data for each subset. The latter assumption is a severe restriction on the time resolution of the reconstruction, since reconstructing the scanned object per subset implies the acquisition of sufficient projection data per subset in order to avoid limited data reconstruction artifacts, which in turn implies that the acquisition time per subset is far from negligible. This limits the applicability of these techniques, as they cannot be applied to reconstruction setups where objects are subject to structural, discontinuous changes that happen in a faster time frame than the acquisition time needed for each subset. This type of changes occurs in a wide variety of reconstruction problems, e.g., the scanning of beam-sensitive 
samples, where regions in the object are damaged by the $\mathrm{X}$-ray beam [15], microstructural investigations of solidification [16] and problems in the field of non-destructive testing, where sudden discontinuous cracks are formed inside the object [10], [17].

A straightforward dynamic imaging acquisition method that employs the subset-reconstruction technique, is an acquisition in which the source and detector rotate in a circular orbit multiple times around the object. In each time window for which the full angular range is covered, a 3D image is reconstructed. This approach is extensively used in medical imaging to obtain real-time reconstructions [18]. The same scanning technique could, however, be used in $\mu \mathrm{CT}$, synchrotron tomography and other advanced lab CT setups as well. In such an approach, it is assumed that the object remains stationary during each time window that covers the full angular range. Evidently, this time window also defines the temporal resolution. Increasing the temporal resolution (by decreasing the number of projection angles or by decreasing the radiation exposure time needed to acquire projection data for one angular direction) will result in reconstructions of poor quality. This limits the temporal resolution for such a subset-reconstruction method from a hardware point of view. From a computational point of view, however, there is still room for improvement, as is illustrated by the techniques introduced in this paper.

In many problems where structural changes complicate the reconstruction process, there is redundancy between projection data acquired at different time points. This redundancy is exploited by the techniques introduced in this paper; we propose an iterative method that generates accurate reconstructions using limited projection data by assuming the existence of regions inside the object that remain constant over time. Since less projection data is needed, time resolution increases. Regularization methods are less suitable for handling structural changes, as they assume that every region in a timevarying object is similar over time, which is certainly not the case for structurally changing objects. Unlike regularization methods, the proposed method enforces similarity by combining iterative update steps over different projection-subset reconstructions. Also, in contrast to the approach introduced in [14], the proposed method does not depend on the prior knowledge of a high quality image of the object before the structural changes initiated. In preliminary work, a regionbased SIRT (rSIRT) algorithm was developed, where these stationary regions are assumed to be known [19]. In this contribution, the rSIRT algorithm is improved and incorporated in an optimization routine that automatically determines the stationary regions inside the object. The developed method was validated on simulation data and on data obtained from a controlled experimental $\mu \mathrm{CT}$ setup. Results show that the proposed method reduces the number of projections and thus a significant increase in time resolution is achieved.

In section II, a brief introduction to algebraic reconstruction methods and computed tomography of static and dynamic objects is given. In Section III, the improved version of the rSIRT algorithm is introduced, together with the iterative routine that incorporates the rSIRT algorithm in a projection distance minimization scheme that automatically estimates

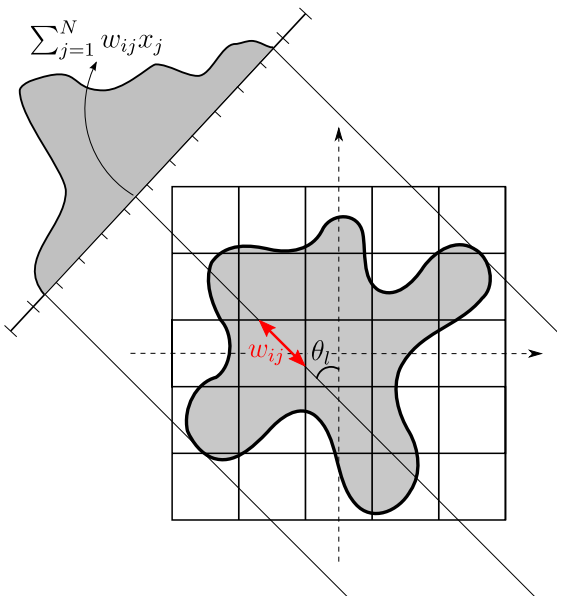

Fig. 1. Illustration of the projection process.

the parameters defining the stationary region. Simulation and experimental results are reported in Section IV. The paper is concluded in Section V.

\section{NOTATION AND CONCEPTS}

In this section, we introduce some basic concepts in computed tomography; more details can be found in [20] and [21]. Next, a description of the SIRT algorithm is given and the concepts of computed tomography are generalized from static objects to dynamic objects, i.e., objects that change over time.

\section{A. Tomography Model}

The reconstructed image of the scanned object is represented on a pixel grid of size $N$. The pixel values of the image are represented by an $N \times 1$ column vector $\boldsymbol{x}=\left(x_{i}\right) \in \mathbb{R}^{N}$. Denote the number of detector elements by $d$ and the total number of projection angles by $n_{\theta}$. Then the scanning process results in $M=n_{\theta} d$ data values, which are log-corrected and ordered in a vector $\boldsymbol{p}=\left(p_{i}\right) \in \mathbb{R}^{M}$. We refer to $\boldsymbol{p}$ as the projection data. Starting from a reconstructed image $\boldsymbol{x}$, the $i^{\text {th }}$ projection value can be simulated by the linear combination $\sum_{j=1}^{N} w_{i j} x_{j}$, where $w_{i j}$ usually represents the intersection length between pixel $j$ and projection line $i$, as is illustrated in Fig. 1. Other methods for determining the weights $w_{i j}$ are also possible (e.g., a strip kernel [20], Joseph's method [22]). For reconstructing 3D volumes from 2D projections, other approaches for simulating the projection values are more suitable, including distance-driven methods [23] and separable footprints [24]. If all the weights are properly stored into a (sparse) matrix $\boldsymbol{W}=\left(w_{i j}\right) \in \mathbb{R}^{M \times N}$, the correspondence between the projection data $\boldsymbol{p}$ and the image $\boldsymbol{x}$ can be written as a system of linear equations

$$
\boldsymbol{W} \boldsymbol{x}=\boldsymbol{p} .
$$

Since noise and discretization effects render Eq.(1) inconsistent, algebraic methods typically minimize the projection distance $\|\boldsymbol{W} \boldsymbol{x}-\boldsymbol{p}\|$ for some norm $\|\cdot\|$.

\section{B. SIRT}

The methods introduced in this paper are based on the Simultaneous Iterative Reconstruction Technique 
(SIRT) [25]. SIRT is known to converge to a solution of $\operatorname{argmin}_{\boldsymbol{x}}\left(\|\boldsymbol{W} \boldsymbol{x}-\boldsymbol{p}\|_{\boldsymbol{R}}^{2}\right)$, where $\boldsymbol{R}=\left(r_{i j}\right) \in \mathbb{R}^{M \times M}$ is the diagonal matrix with inverse row sums of the projection matrix $\boldsymbol{W}$; its diagonal elements are given by $r_{i i}=1 / \sum_{j} w_{i j}$. Define $C=\left(c_{i j}\right) \in \mathbb{R}^{N \times N}$ as the diagonal matrix with the inverse column sums of $\boldsymbol{W}$ (i.e., $c_{j j}=1 / \sum_{i} w_{i j}$ ). Then, the update expression for SIRT can be compactly written as

$$
\boldsymbol{x}^{(k+1)}=\boldsymbol{x}^{(k)}+\boldsymbol{C} \boldsymbol{W}^{T} \boldsymbol{R}\left(\boldsymbol{p}-\boldsymbol{W} \boldsymbol{x}^{(k)}\right) .
$$

\section{Dynamic Objects}

A slightly different modeling approach is needed when the scanned object changes over time, as we can no longer assume the object to be the same for every projection angle $\theta_{l}\left(l \in\left\{1, \ldots, n_{\theta}\right\}\right)$. Hence, this assumption is discarded and replaced by the assumption that the object remains stationary (i.e., it does not move or change) during the acquisition of tomographic data corresponding to a single projection angle. The time-varying object is therefore represented as a series of subsequent vectors $\boldsymbol{x}_{l} \in \mathbb{R}^{N}$, where $l \in\left\{1, \ldots, n_{\theta}\right\}$. We refer to the $i^{\text {th }}$ pixel in the reconstruction $\boldsymbol{x}_{l}$ at time index $l$ as $\boldsymbol{x}_{l}(i)$. Define $\boldsymbol{W}_{l} \in \mathbb{R}^{d \times N}$ as the submatrix of $\boldsymbol{W}$ that corresponds to the projection angle $\theta_{l}$ and $\boldsymbol{p}_{l} \in \mathbb{R}^{d}$ as the subvector of the projection data $\boldsymbol{p}$ corresponding to the same angle $\theta_{l}$. Then the reconstruction $\boldsymbol{x}_{l}$ at time index $l$ should ideally satisfy $\boldsymbol{W}_{l} \boldsymbol{x}_{l}=\boldsymbol{p}_{l}$. These equations can be written in a single matrix equation

$$
\left[\begin{array}{cccc}
\boldsymbol{W}_{1} & 0 & \cdots & 0 \\
0 & \boldsymbol{W}_{2} & & 0 \\
\vdots & & \ddots & \vdots \\
0 & 0 & \cdots & \boldsymbol{W}_{n_{\theta}}
\end{array}\right]\left[\begin{array}{c}
\boldsymbol{x}_{1} \\
\boldsymbol{x}_{2} \\
\vdots \\
\boldsymbol{x}_{n_{\theta}}
\end{array}\right]=\tilde{\boldsymbol{W}} \tilde{\boldsymbol{x}}=\boldsymbol{p}
$$

where $\tilde{\boldsymbol{W}}$ represents the block diagonal matrix consisting of blocks $\boldsymbol{W}_{1}, \boldsymbol{W}_{2}, \ldots, \boldsymbol{W}_{n_{\theta}}$ and $\tilde{\boldsymbol{x}} \in \mathbb{R}^{n_{\theta} N}$ represents the vertical concatenation of the vectors $\boldsymbol{x}_{1}, \boldsymbol{x}_{2}, \ldots, \boldsymbol{x}_{n_{\theta}}$.

\section{MEthods}

In this section, we introduce the rSIRT algorithm, a modified version of the well known SIRT algorithm that combines traditional SIRT update steps over different regions and points in time. Next, a formal definition of the scanning protocol is given. The rSIRT algorithm assumes that stationary regions within the object are given a priori. As in practice, this assumption does not hold, we propose a methodology to automatically estimate the stationary regions: a B-spline model for region description, together with the rSIRT algorithm, are incorporated in a weighted projection distance minimization scheme, that automatically calculates the parameters describing the stationary regions.

\section{A. $r S I R T$}

The scheme to calculate a single rSIRT iteration is displayed in the flowchart of Fig. 2. The phantom of Fig. 5(a)-(d) is used for visualization purposes. We assume the scanned object to consist of stationary regions and regions that change over

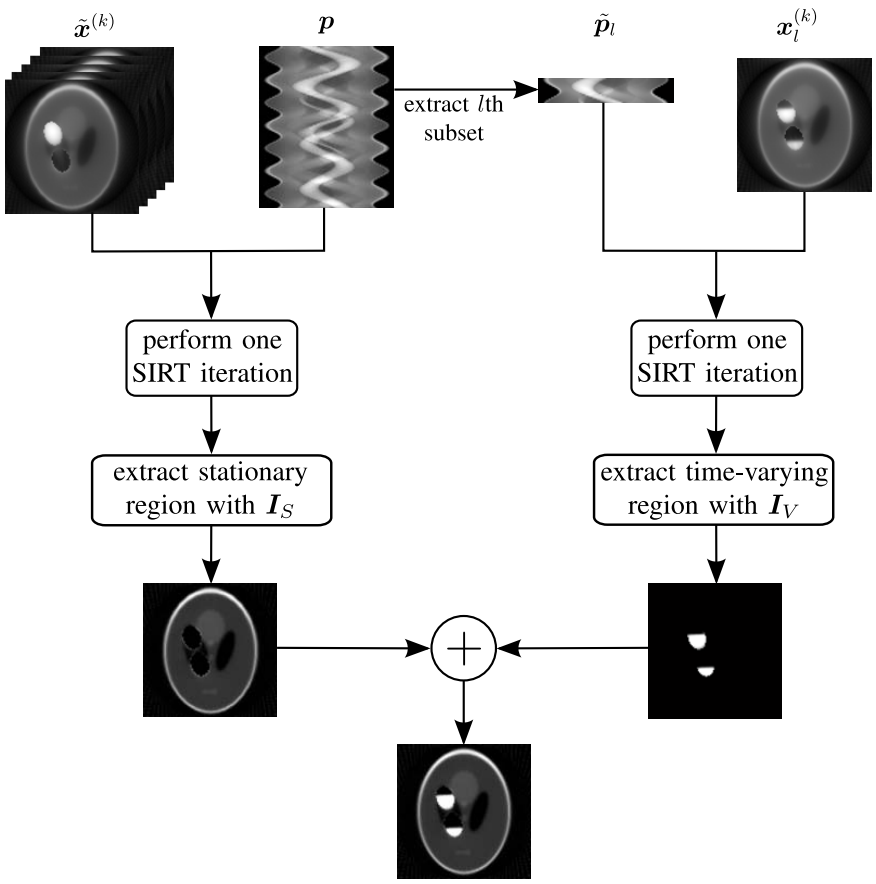

Fig. 2. Flowchart of a single rSIRT iteration. Starting from a previous estimate $\tilde{\boldsymbol{x}}^{(k)}$ and the projection data $\boldsymbol{p}$, the rSIRT algorithm calculates for every time index $l$ a new estimate $\boldsymbol{x}_{l}^{(k+1)}$.

time, i.e., variable regions. Define $S \subset\{1, \ldots, N\}$ as the set of pixel indices that correspond to the stationary regions and define $V$ as its complement, i.e., $V=\{1, \ldots, N\} \backslash S$ is the set of pixel indices corresponding to the object's variable regions. Let $\boldsymbol{I}_{V} \in\{0,1\}^{N \times N}$ be the binary diagonal matrix representing the operator that sets all pixels belonging to the stationary region to 0 . Its diagonal elements are given by

$$
\boldsymbol{I}_{V}(j, j)= \begin{cases}1 & \text { if } j \in V \\ 0 & \text { otherwise }\end{cases}
$$

Define the binary diagonal matrix $\boldsymbol{I}_{S} \in\{0,1\}^{N \times N}$ analogously. It is the operator setting all pixels in the variable regions to 0 . Define a window size $n_{w} \in \mathbb{N} \backslash\{0\}$ and use this number to define the projection matrix

$$
\tilde{\boldsymbol{W}}_{l}=\left[\begin{array}{c}
\boldsymbol{W}_{l-\left\lfloor n_{w} / 2\right\rfloor} \\
\boldsymbol{W}_{l-\left\lfloor n_{w} / 2\right\rfloor+1} \\
\vdots \\
\boldsymbol{W}_{l+\left\lceil n_{w} / 2\right\rceil-1}
\end{array}\right]
$$

for each time index $l$ with $\left\lfloor n_{w} / 2\right\rfloor+1 \leq l \leq n_{\theta}-\left\lceil n_{w} / 2\right\rceil+1$. The matrix $\tilde{\boldsymbol{W}}_{l} \in \mathbb{R}^{n_{w} d \times N}$ represents the projection operator that projects along the $n_{w}$ directions centered around projection angle $\theta_{l}$. Define

$$
\tilde{\boldsymbol{W}}_{l}=\left[\begin{array}{c}
\boldsymbol{W}_{1} \\
\vdots \\
\boldsymbol{W}_{n_{w}}
\end{array}\right]
$$


for $l<\left\lfloor n_{w} / 2\right\rfloor+1$ and

$$
\tilde{\boldsymbol{W}}_{l}=\left[\begin{array}{c}
\boldsymbol{W}_{n_{\theta}-n_{w}+1} \\
\vdots \\
\boldsymbol{W}_{n_{\theta}}
\end{array}\right]
$$

for $l>n_{\theta}-\left\lceil n_{w} / 2\right\rceil+1$. Next, let $\tilde{\boldsymbol{p}}_{l} \in \mathbb{R}^{n_{w} d}$ denote the projection data corresponding to the projection directions as they are encoded in $\tilde{\boldsymbol{W}}_{l}$. Finally, define $\tilde{\boldsymbol{R}}_{l} \in \mathbb{R}^{n_{w} d \times n_{w} d}$ as the diagonal matrix with inverse row sums of $\tilde{\boldsymbol{W}}_{l}$ and $\tilde{\boldsymbol{C}}_{l} \in \mathbb{R}^{N \times N}$ as the diagonal matrix with inverse column sums of $\tilde{\boldsymbol{W}}_{l}$. The introduced notations allow us to describe the rSIRT algorithm as the following iterative process:

$$
\begin{aligned}
\boldsymbol{x}_{l}^{(k+1)}= & \boldsymbol{x}_{l}^{(k)}+\boldsymbol{I}_{S} \boldsymbol{C} \boldsymbol{W}^{T} \boldsymbol{R}\left(\boldsymbol{p}-\tilde{\boldsymbol{W}} \tilde{\boldsymbol{x}}^{(k)}\right) \\
& +\boldsymbol{I}_{V} \tilde{\boldsymbol{C}}_{l} \tilde{\boldsymbol{W}}_{l}^{T} \tilde{\boldsymbol{R}}_{l}\left(\tilde{\boldsymbol{p}}_{l}-\tilde{\boldsymbol{W}}_{l} \boldsymbol{x}_{l}^{(k)}\right) .
\end{aligned}
$$

This update needs to be calculated for every $l \in\left\{1, \ldots, n_{\theta}\right\}$ before incrementing the iteration count $k$. Basically, Eq. (7) calculates two update steps. A traditional SIRT update step for the stationary region using all available projection data $\boldsymbol{p}$, corresponding to the left hand side of the flowchart in Fig. 2, and an update step for the variable region using only the projection data centered around the current time index $l$, corresponding to the right hand side of the flowchart in Fig. 2.

The rSIRT algorithm with initial estimate $\tilde{\boldsymbol{x}}^{(0)}=\mathbf{0}$ is representable as a linear operator applied to the projection data $\boldsymbol{p}$, which we denote as $\tilde{\boldsymbol{x}}^{(K)}=\boldsymbol{S}_{K} \boldsymbol{p}$, where $K$ denotes the number of rSIRT iterations. This can be proven in an analogous manner to the proof in [26], where the regular SIRT algorithm is proven to be a linear operator on the projection data. The details of this derivation can be found in Appendix C.

Note that for a practical implementation, the update term in Eq. (7) for the stationary region $\boldsymbol{I}_{S} \boldsymbol{C} \boldsymbol{W}^{T} \boldsymbol{R}\left(\boldsymbol{p}-\tilde{\boldsymbol{W}} \tilde{\boldsymbol{x}}^{(k)}\right)$ needs to be calculated only once, since it is exactly the same for every time index $l$. Also, instead of working with matrices, a ray-based approach can be used for the forward and backward projection operators [27].

\section{B. Scanning Protocol}

It is well known that image reconstruction from a limited angular range deteriorates the reconstruction quality [28]. Hence, we need projection data per time point that has a sufficiently large angular range, since the goal is to reconstruct the object at different time points. This is why source and detector are rotated multiple times around the object, acquiring projection data at $n_{w}$ angular directions approximately every 180 degrees. Define an angular stepsize $\theta_{\triangle}=\pi / n_{w}$ and choose the first equiangularly spaced $n_{w}$ projection directions as $\theta_{i}=(i-1) \theta_{\triangle} \in\left[0, \pi-\theta_{\triangle}\right]$ for $i=1, \ldots, n_{w}$. The other projection directions are defined as

$$
\theta_{k n_{w}+i}=k\left(\pi-\theta_{\triangle}\right)+k \frac{\pi}{n_{\theta}}+(i-1) \theta_{\triangle}
$$

for $i=1, \ldots, n_{w}, k=1, \ldots,\left\lfloor n_{\theta} / n_{w}\right\rfloor$ and $k n_{w}+i \leq n_{\theta}$. The angles $\theta_{1}, \theta_{2}, \ldots, \theta_{2 n_{w}}$ are schematically displayed in Fig. 3.

The small incremental step $k \frac{\pi}{n_{\theta}}$ in Eq. (8) ensures that there exist no two projection directions that are equal modulo $\pi$.

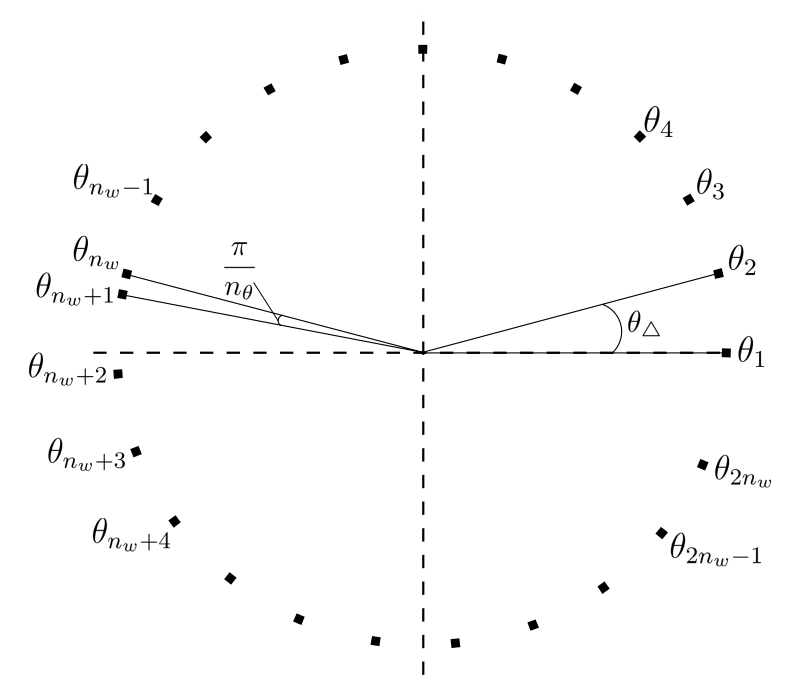

Fig. 3. Source positions for the first $2 n_{w}$ projection directions indicated by square markers.

On the one hand, using the same projection angle (modulo $\pi$ for parallel beam and modulo $2 \pi$ for fan beam) more than once increases the signal-to-noise ratio (SNR). On the other hand, this will introduce redundant angular information, since the rays going through the stationary region give the same projection values (up to noise). This situation is avoided by choosing the projection angles via Eq. (8). Furthermore, Eq. (8) guarantees that each subsequent $n_{w}$ projection directions cover a range of approximately 180 degrees, thus avoiding limited view artifacts.

\section{Region Inconsistency Minimization}

In this section, we introduce a projection distance minimization scheme that uses the rSIRT algorithm to estimate the stationary region automatically.

For describing the variable region, a B-spline based closed curve model is used. More details can be found in Appendix A. The coordinates of the B-spline closed curve control points are ordered in a parameter vector $\boldsymbol{\alpha}$, describing the degrees of freedom of our optimization routine. Using this region model, we present a measure that indicates how likely a stationary region is to occur. The measure itself is introduced first, while its different components are explained later. The measure is named region Inconsistency $(r I)$ and is defined as

$$
r I_{\lambda, \mu_{1}, \mu_{2}}(\boldsymbol{\alpha})=\left\|\tilde{\boldsymbol{W}} \boldsymbol{S}_{K}^{\boldsymbol{\alpha}} \boldsymbol{p}-\boldsymbol{p}\right\|_{\overline{\boldsymbol{R}}_{\boldsymbol{\alpha}}^{\lambda}}^{2}+\mu_{1} P_{1}(\boldsymbol{\alpha})+\mu_{2} P_{2}(\boldsymbol{\alpha})
$$

The first term in Eq. (9) is a data fidelity term. Its calculation is displayed schematically in the uppermost part of the flowchart in Fig. 4. Since the linear operator describing $K$ iterations of rSIRT depends on the variable region's shape, we denote it by $S_{K}^{\alpha}$. The rSIRT reconstruction $\boldsymbol{S}_{K}^{\alpha} \boldsymbol{p}$ is forward projected with the projection matrix $\tilde{\boldsymbol{W}}$ and compared to the original data $\boldsymbol{p}$ through the norm $\|\cdot\|_{\overline{\boldsymbol{R}}_{\alpha}^{\lambda}}^{2}$. Instead of weighting this norm with the classical inverse row sum matrix $\boldsymbol{R}$ (see Section II-B), it is weighted with a normalized version of an adjusted inverse row sum diagonal matrix $\boldsymbol{R}_{\boldsymbol{\alpha}}^{\lambda}$, where the ray intersection lengths 


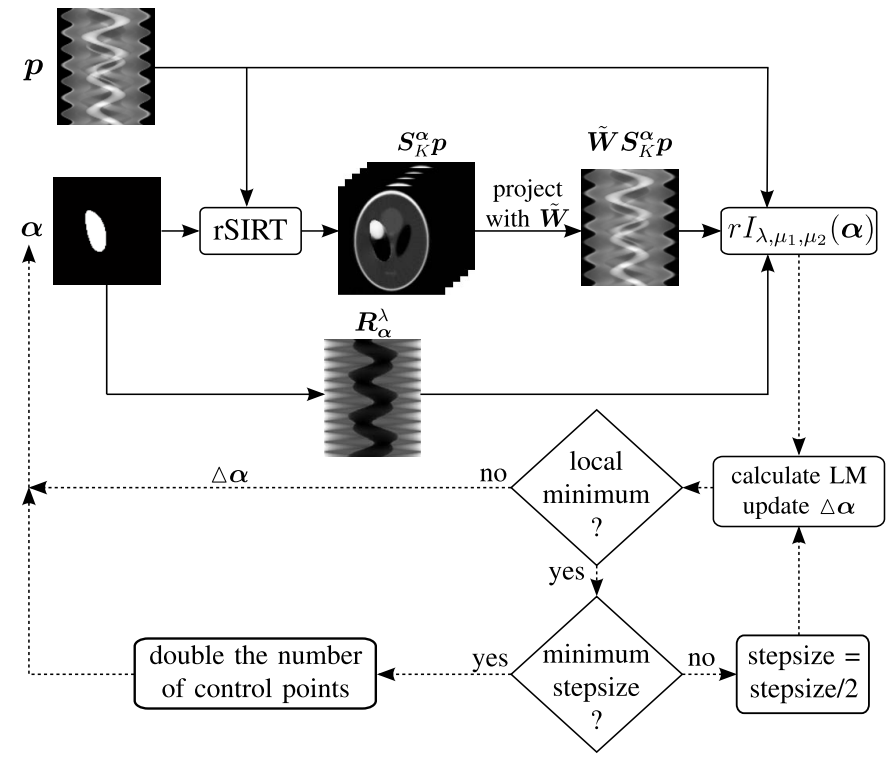

Fig. 4. Flowchart of the optimization strategy. The solid arrows indicate the calculation of the region inconsistency, while the dotted arrows illustrate the optimization strategy.

through pixels belonging to the variable region are multiplied with a factor $\lambda>1$. Hence the diagonal elements of $\boldsymbol{R}_{\boldsymbol{\alpha}}^{\lambda}$ are defined as

$$
\boldsymbol{R}_{\boldsymbol{\alpha}}^{\lambda}(i, i)=1 / \sum_{j}\left(\left(1+\boldsymbol{I}_{V}(j, j)(\lambda-1)\right) w_{i j}\right) .
$$

By normalizing $\boldsymbol{R}_{\boldsymbol{\alpha}}^{\lambda}$ such that it has the same mean along its diagonal as $\boldsymbol{R}$, the normalized diagonal matrix $\overline{\boldsymbol{R}}_{\boldsymbol{\alpha}}^{\lambda}$ is formed. Basically, this weight matrix reflects the higher confidence in measurements corresponding to rays that mainly intersected the stationary region. A more detailed description of $\overline{\boldsymbol{R}}_{\boldsymbol{\alpha}}^{\lambda}$ can be found in Appendix B. The term $P_{1}(\boldsymbol{\alpha})$ in Eq. (9) gives a penalty to self-intersecting curves; it simply counts the number of times the curve intersects itself. The term $P_{2}(\boldsymbol{\alpha})$ gives a penalty to regions that exceed the boundaries of the reconstruction domain. It is given by the sum of the Euclidean distances of each control point outside the reconstruction domain to the closest point inside the reconstruction domain, which is assumed to be confined within $[-1,1] \times[-1,1]$. The severity of the penalties defined by $P_{1}(\boldsymbol{\alpha})$ and $P_{2}(\boldsymbol{\alpha})$ are controlled by the parameters $\mu_{1}$ and $\mu_{2}$.

An adapted variant of the Levenberg-Marquardt (LM) algorithm [29] is used for minimizing $r I$, which is illustrated in the lower part of the flowchart in Fig. 4. The $r I$ function is a non-convex function that, due to noise and discretization effects, has a non-smooth, coarse landscape on a small scale. This coarseness can make the finite difference approximation of the objective function's gradient inaccurate. Therefore, finite differences are calculated with a parameter increment (referred to as the stepsize), starting from a larger initial stepsize that is halved every time the solver reaches a local minimum. When the stepsize becomes smaller than a specified threshold, the stepsize is reinitialized and the number of spline control points is doubled by applying Boehm's formula for knot insertion [30], thus providing more degrees of freedom to

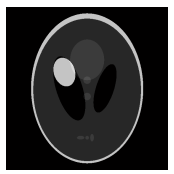

(a) $t=t_{1}$

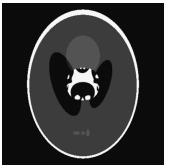

(e) $t=t_{1}$

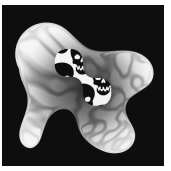

(i) $t=t_{1}$

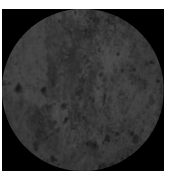

(m) $t=t_{1}$

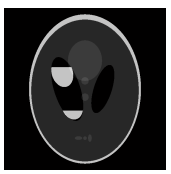

(b) $t=t_{120}$

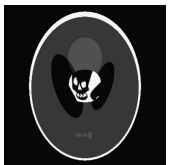

(f) $t=t_{120}$

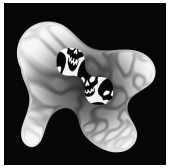

(j) $t=t_{120}$

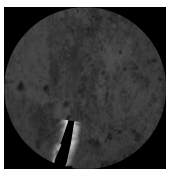

(n) $t=t_{120}$

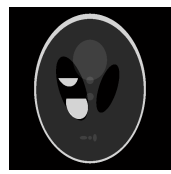

(c) $t=t_{180}$

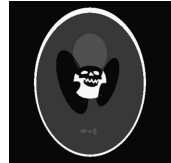

(g) $t=t_{180}$

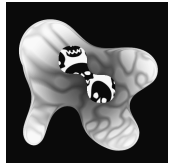

(k) $t=t_{180}$

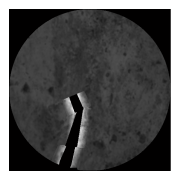

(o) $t=t_{180}$

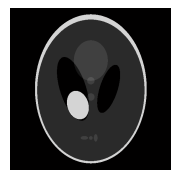

(d) $t=t_{300}$

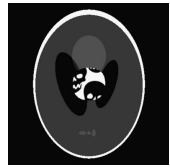

(h) $t=t_{300}$

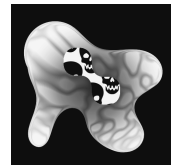

(1) $t=t_{300}$

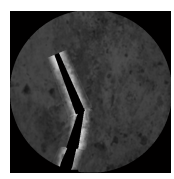

(p) $t=t_{300}$
Fig. 5. Each row represents a different phantom. Each column represents a different point in time. (a-d) Phantom 1: a modified Shepp-Logan phantom. The changes in the phantom resemble a liquid flowing from one chamber of the object into another. (e-h) Phantom 2: a modified Shepp-Logan phantom. In the middle of the phantom a structure with skulls is changing over time. (i-1) Phantom 3: blob-shaped phantom with an eight-like variable region in the middle. (m-p) Phantom 4: image of material in which a crack is formed.

the solver by this multi-resolution approach on the parameter vector $\boldsymbol{\alpha}$.

\section{EXPERIMENT}

In this section, rSIRT and the region inconsistency minimization are validated on numerical and experimental data.

\section{A. Numerical Simulations}

Consider the phantoms in Fig.5. Phantom 1 is displayed in Fig. 5(a)-(d). It is a modified Shepp-Logan phantom. The phantom resembles a sample in which fluid flows from one chamber into another during the acquisition of the CT data. Phantom 2 [Fig. 5(e)-(h)] and phantom 3 [Fig. 5(i)-(1)] are artificial phantoms in which a structurally varying region in the form of a circle and an eight-like shape are present in the object. Finally, phantom 4, displayed in Fig. 5(m)-(p), represents an object in which a crack is formed.

Projections were simulated with the scanning protocol of Section III-B where we took the window size to be $n_{w}=30$. We simulated 300 projections using a strip kernel and a higher resolution version of the phantom, i.e., on a $500 \times 500$ isotropic pixel grid, while the algorithm calculates reconstructions on a $100 \times 100$ isotropic pixel grid. Also, Poisson distributed noise was applied to the projection data assuming an incoming beam intensity of 10000 (photon count) per detector pixel. 


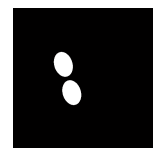

(a)

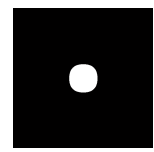

(b)

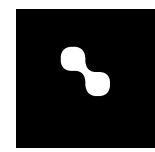

(c)

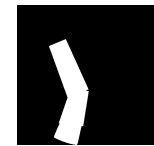

(d)

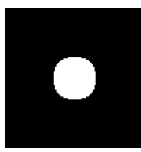

(e)
Fig. 6. (a)-(d) Ground truth for the time-varying region for all simulation phantoms. (e) Initial estimate for simulation experiments with the region inconsistency minimization algorithm. In all figures, white pixels belong to the time-varying region and black pixels belong to the stationary region.

TABLE I

RMSE After 100 Iterations For All Simulated Phantoms (ROWS) AND FOR DIFFERENT METHODS (COLUMNS)

\begin{tabular}{c|c|c|c|c} 
& SIRT & Conventional & rSIRT & rSIRT-opt \\
\hline \hline Phantom 1 & 0.13379 & 0.12291 & 0.09767 & 0.09793 \\
\hline Phantom 2 & 0.12508 & 0.13702 & 0.11247 & 0.11257 \\
\hline Phantom 3 & 0.12993 & 0.13627 & 0.11038 & 0.11068 \\
\hline Phantom 4 & 0.36335 & 0.36543 & 0.28545 & 0.28867
\end{tabular}

For validation, we used three different measures. A first figure of merit is the Root Mean Square Error (RMSE). Denote the calculated reconstruction by $\hat{\boldsymbol{x}}$ and the phantom used to generate the data by $\tilde{\boldsymbol{x}}$. Then the RMSE is given by

$$
R M S E=\sqrt{\left\langle(\hat{\boldsymbol{x}}-\tilde{\boldsymbol{x}})^{2}\right\rangle},
$$

where the squaring should be interpreted pointwise and where $\langle\cdot\rangle$ represents the average. We upsampled the calculated reconstruction 5 times in order to perform the RMSE calculations on the $500 \times 500$ grid. A second measure is the projection distance, defined as

$$
P D=\|\tilde{\boldsymbol{W}} \hat{\boldsymbol{x}}-\boldsymbol{p}\|_{2} .
$$

Finally, for the validation of the region inconsistency minimization, we also look at the relative Number of Misclassified Pixels (rNMP), i.e., the number of misclassified variable region pixels (with respect to the ground truth variable region) divided by the total number of pixels belonging to the ground truth variable region. The rNMP was also calculated on the original $500 \times 500$ grid by scaling up the optimized region.

The performance of rSIRT was compared to two other reconstruction methods. A first method is the conventional method. It calculates a regular SIRT reconstruction per subset of the projection data $\tilde{\boldsymbol{p}}_{l}$ with the corresponding projection matrices as they are defined in Eq.(4), Eq. (5) and Eq. (6). Also, a regular SIRT reconstruction was used for comparison, i.e., a SIRT reconstruction using all the projection data $p$ where the object was regarded as stationary through time (see Section II-B).

First, a validation of the rSIRT algorithm is presented. Since the phantoms are simulated, the stationary region is known and can be used as prior knowledge for rSIRT. The ground truth regions are displayed in Fig. 6(a)-(d).

The RMSE after 100 iterations with an initial zero estimate was calculated for all phantoms and for each of the reconstruction methods. The results are summarized in the columns "SIRT", "Conventional" and " $r S I R T$ " in Table I. In this table, the column $r S I R T$ refers to the rSIRT reconstruction with

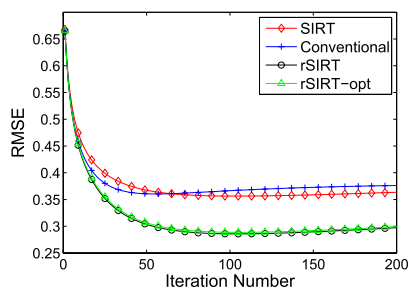

(a)

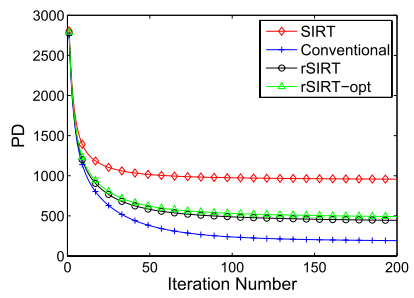

(b)
Fig. 7. The convergence of the different methods for phantom 4. (a) RMSE as a function of iteration number. (b) PD as a function of iteration number.

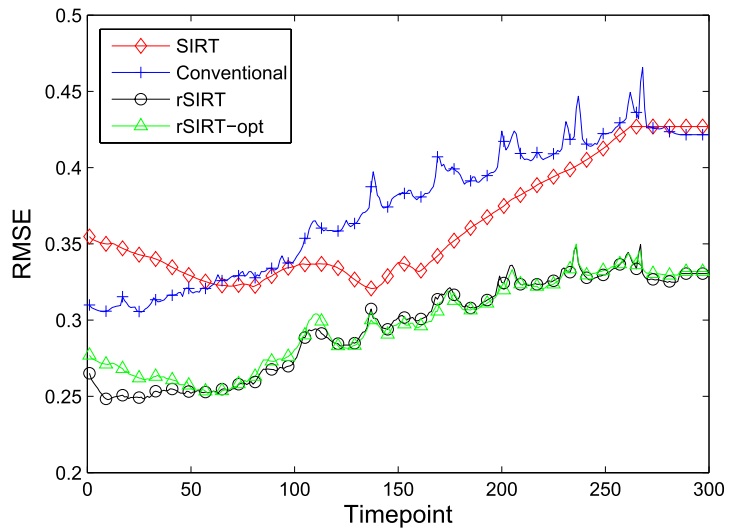

Fig. 8. RMSE per point in time for reconstructions after 100 iterations for phantom 4.

the ground truth stationary region. In order to illustrate the convergence properties of rSIRT, more detailed plots of the results for phantom 4 are presented in Figs. 7 and 8. Fig. 7(b) indicates that all methods reduce the projection distance as the iteration number increases. As the reconstruction per time point for the conventional method must only match with a subset of all projection data, its projection distance decreases the fastest. However, since the RMSE assesses the image quality directly, it can be concluded from Fig. 7(a) that rSIRT has superior performance. Also, SIRT is unable to capture the object's dynamics, as it generates one reconstruction for the entire projection data set. The conventional method can capture some dynamics, but reconstruction quality is severely degraded. The rSIRT algorithm combines the better properties of the conventional method and SIRT, and reconstructs the object with improved image quality and time resolution. The RMSE at each time instance of the reconstructions after 100 iterations is displayed in Fig. 8, confirming the previous statements.

Also, the region inconsistency minimization was validated on the simulated data. The parameter setup was $\lambda=10$, spline degree $k=2, \mu_{1}=1, \mu_{2}=1, n_{w}=30$ and $K=30$. The initial estimate for the variable region is displayed in Fig. 6(e). Other initial estimates (e.g., not intersecting with the ground truth variable region) may affect convergence speed, but typically produce the same result. The number of control points was doubled three times, resulting in a final region descriptor of 32 control points. In the last optimization round, i.e., when using 32 control points, the number of rSIRT iterations $K$ was set to 60 to obtain a more accurate 


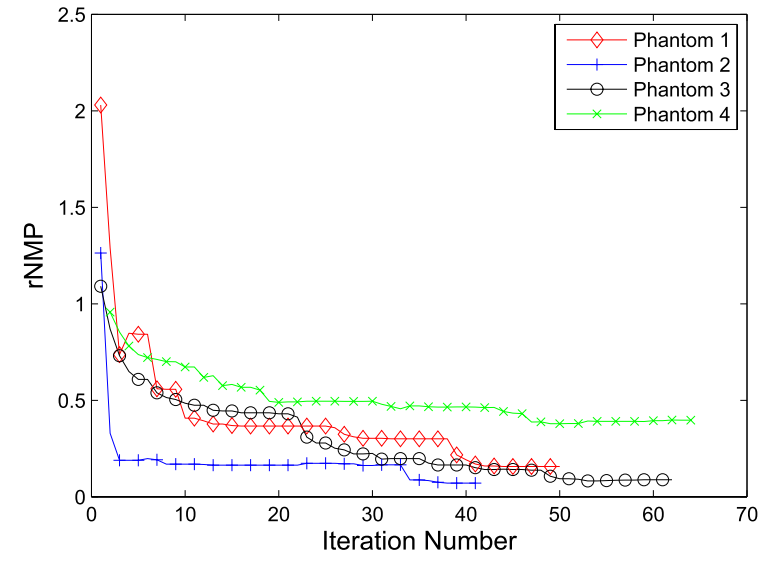

Fig. 9. rNMP as a function of iteration number of the region inconsistency minimization algorithm.

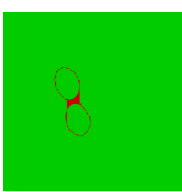

(a) Phantom $\mathrm{rNMP}=0.157$
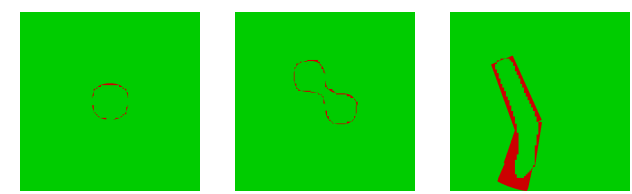

(b) Phantom 2, (c) Phantom 3, (d) Phantom 4,

Fig. 10. Region estimate after region inconsistency minimization. Red indicates misclassified pixels, green indicates correctly classified pixels. The corresponding rNMP is also indicated for every region estimate.

result. The output of the region inconsistency minimization algorithm — the stationary region — was used as input for the rSIRT algorithm with 100 iterations. The RMSE of this rSIRT reconstruction is tabulated in the column " $r S I R T$-opt" in Table I. The rNMP as a function of iteration number is displayed in Fig. 9. The resulting variable region estimates and its misclassified pixels are visualized in Fig. 10.

These results indicate that the region inconsistency minimization algorithm is able to estimate a stationary region that closely approximates the ground truth stationary region, resulting in an almost identical RMSE for the rSIRT reconstruction based on the estimated region and the ground truth region. Despite the region estimate for phantom 4 being less accurate [see Fig. 10(d)], Fig. 8 indicates that the RMSE is still significantly improved. Also, Fig. 7(a) illustrates that the convergence properties remain almost unaltered.

The calculation of 30 rSIRT iterations in the experimental setup as described above (i.e., reconstructing on a $100 \times 100$ pixel grid and using projection data consisting of 300 projection angles), takes about 5 seconds with an unoptimized GPU implementation. Depending on the number of iterations needed by the region inconsistency minimization algorithm, it takes about 2-4 hours to calculate a variable region estimate. However, this computational time could be significantly reduced by parallelizing the $r I$ function evaluations, which are needed for the gradient calculation.

\section{B. Experimental Data}

A sequence of cone beam projection images of a plexiglas (Polymethyl Methacrylate) resolution phantom was acquired in a SkyScan $1172 \mu \mathrm{CT}$ scanner. Each radiograph was acquired using a source voltage of $80 \mathrm{kV}$, a source current of $124 \mu \mathrm{A}$,

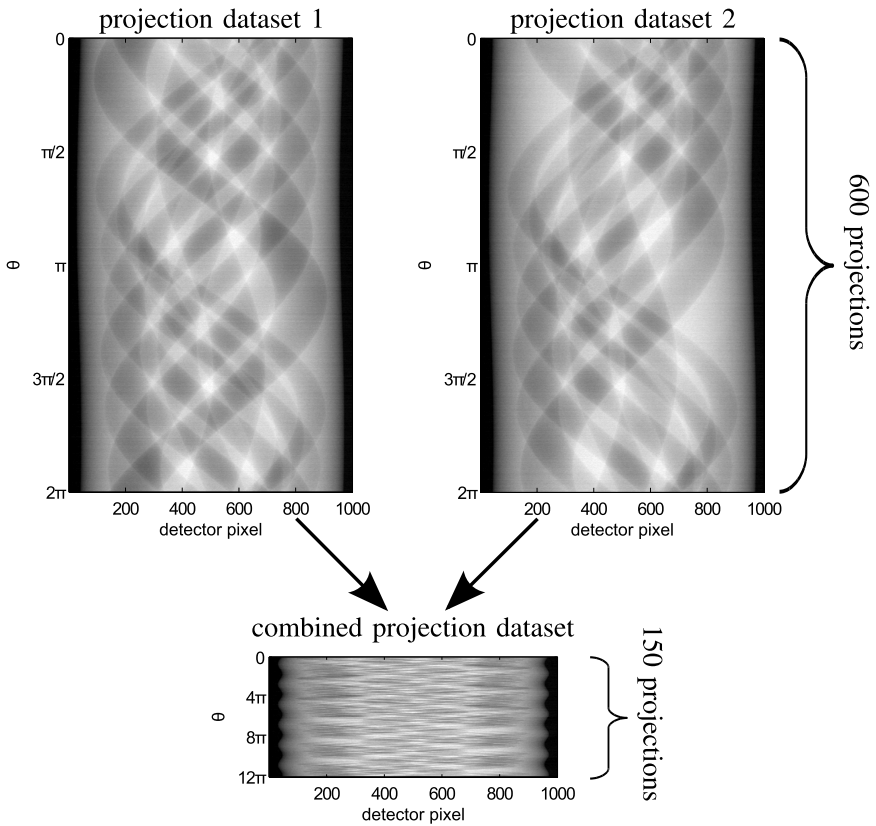

Fig. 11. Illustration of the combination of the two datasets, consisting of 600 projections each, into one single dataset, consisting of only 150 projections.

a $0.5 \mathrm{~mm} \mathrm{Al} \mathrm{filter} \mathrm{and} \mathrm{an} \mathrm{exposure} \mathrm{time} \mathrm{of} 360 \mathrm{~ms}$. The object has been scanned over the full angular range of $360^{\circ}$ with a $0.6^{\circ}$ tilt increment, this resulted in a total of $600 \mathrm{log}$ corrected projection images per scan. To increase the SNR, the original detector pixels of size $9.01 \mu \mathrm{m}$ were downsampled by a factor 4 in both dimensions, resulting in projection images of dimension $666 \times 1000$ with detector pixel size $36.04 \mu \mathrm{m}$. Reconstructions are performed on a $1000 \times 1000$ isotropic pixel grid of the slice on the optical axis. Having a horizontal cone-beam angle of $9.52^{\circ}$ and a source to origin distance of $130.21 \mathrm{~mm}$, this results in a pixel size of $21.69 \mu \mathrm{m}$. Since ringand beam-artifact correction goes beyond the focus of this paper, we have preprocessed the projection images to correct these artifacts using the standard SkyScan NRecon software package.

A cross section on the optical axis of the plexiglas phantom is displayed in Fig. 14(a). The $2 \mathrm{~cm}$ diameter plexiglas cylinder was drilled with three $4 \mathrm{~mm}$ diameter holes, four $3 \mathrm{~mm}$ diameter holes and four $2 \mathrm{~mm}$ diameter holes. We created 2 datasets, each consisting of 600 projection images of the phantom. The first dataset is a regular scan of the phantom. Next, one of the pores was filled with water and the second dataset was acquired with exactly the same parameter setup as for the acquisition of the first dataset. A ground truth for each of the datasets was created by calculating a SIRT reconstruction using 50 iterations and the full set of 600 projections. Subsequently the reconstruction was segmented using the method of Otsu [31]. The obtained ground truth for the first and the second dataset are displayed in Fig. 14(a) and (b), respectively.

We combined the first two projection datasets into a single projection dataset as if the hole was filled with water during the data acquisition. The combination of the projection datasets is illustrated in Fig. 11. 


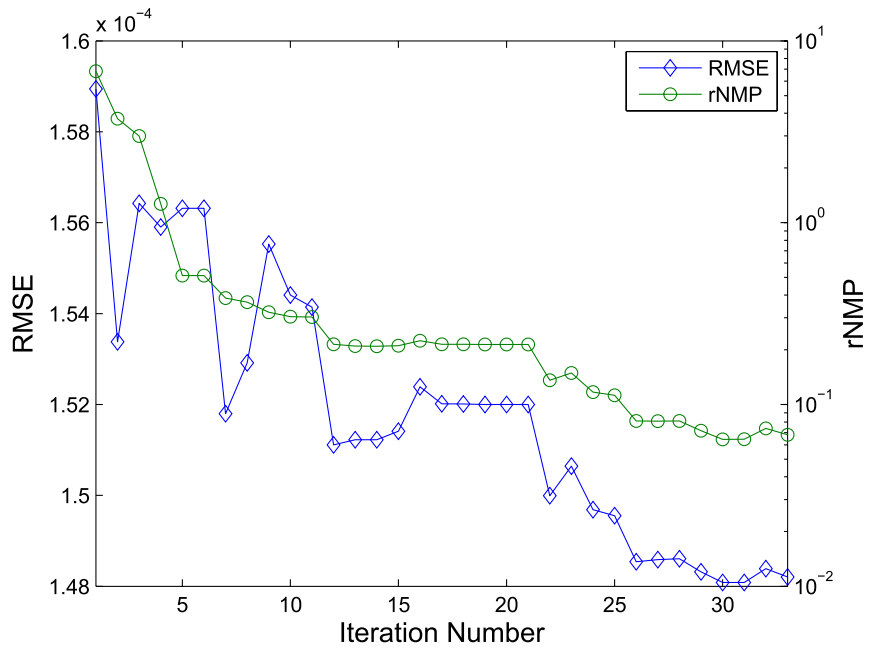

Fig. 12. RMSE (left vertical axis) and rNMP (right vertical axis) in function of iteration number for the region inconsistency minimization applied to the plexiglas resolution phantom. The RMSE values are calculated on the reconstruction generated with 40 rSIRT iterations and the intermediate variable region estimate.

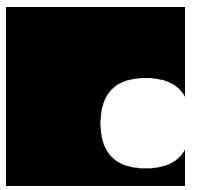

(a) estimate

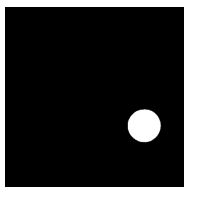

Initial (b) Ground truth

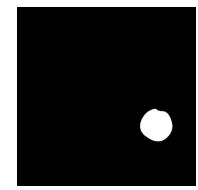
tion result pixels rNMP $=0.0681$ (c) $r I$ minimiza-

Fig. 13. Region inconsistency minimization results. In (a)-(c), the initial estimate, the ground truth and the region inconsistency minimization result are shown, respectively. White pixels indicate the time-varying region and black pixels indicate the stationary region. In (d), the misclassified pixels are visualized, red pixels are misclassified and green pixels are correctly classified.

The first 75 projections were taken from the first dataset, where the projections were ordered analogously to the scanning protocol from section III-B with window size $n_{w}=25$. The only difference is that we made sure that each $n_{w}$ directions correspond to approximately $360^{\circ}$, since each of the projection datasets corresponds to a full $360^{\circ}$ angular range. The next 75 projections were taken from the second dataset, and ordered in the same manner as the first dataset. This resulted in a projection dataset consisting of 150 angular directions, where source and detector have rotated a total of 6 times around the object.

Next we applied the region inconsistency minimization to the resulting combined projection dataset. The used parameters were $\lambda=10$, spline degree $k=2, \mu_{1}=1, \mu_{2}=1, n_{w}=25$ and $K=25$. The initial variable region estimate is displayed in Fig. 13(a). The intermediate variable region estimates, i.e., after each iteration of the region inconsistency minimization algorithm, were used to calculate the rNMP as well as the RMSE of the associated rSIRT reconstruction. These results are displayed in Fig. 12. Convergence was reached after 33 iterations. The final variable region estimate was used to generate an rSIRT reconstruction, which is referred to as "rSIRT-opt." The RMSE of this reconstruction with respect to the ground truth was calculated and compared to the results
TABLE II

RMSE AFter 40 Iterations For the PleXiglas Phantom

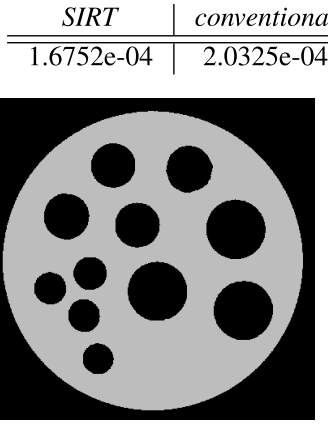

(a) Ground truth at time instance $t_{i}$ with $i \leq 75$

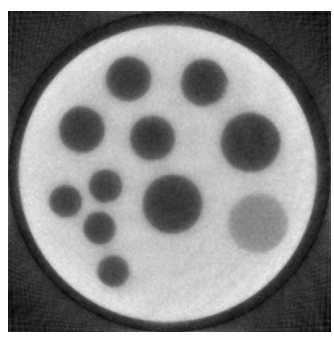

(c) $S I R T-t_{30}$

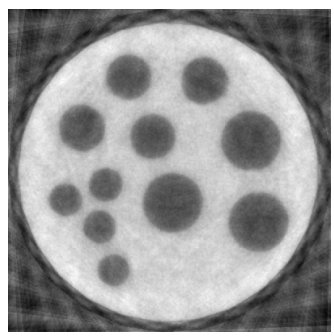

(e) conventional $-t_{30}$

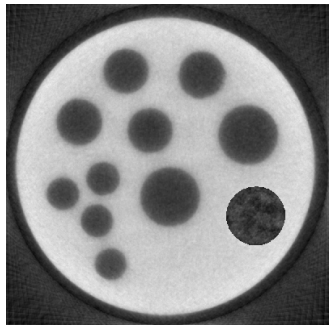

(g) $r S I R T-t_{30}$

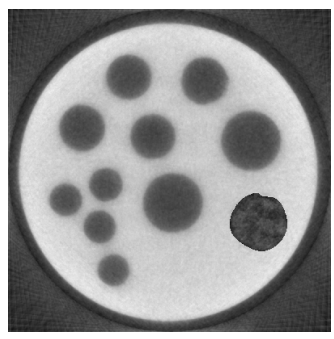

(i) $r S I R T$-opt $-t_{30}$

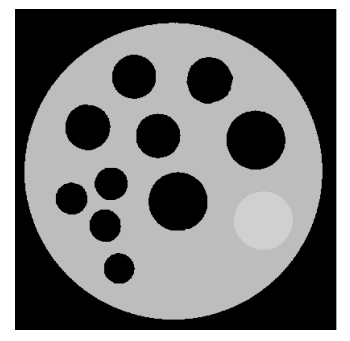
stance $t_{i}$ with $i>75$

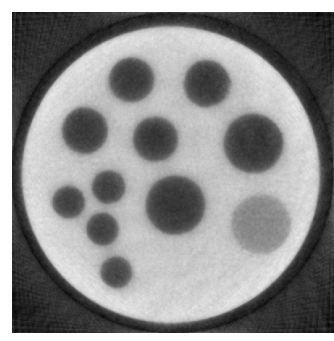

(d) $S I R T-t_{120}$

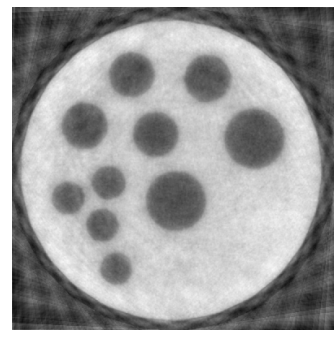

(f) conventional - $t_{120}$

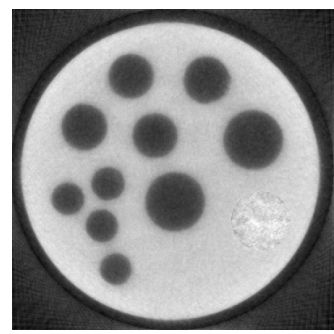

(h) $r S I R T-t_{120}$

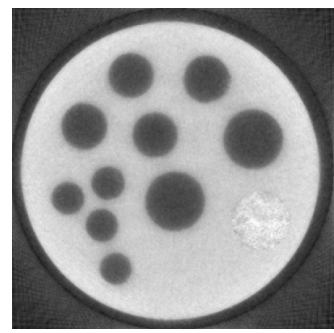

(j) rSIRT-opt $-t_{120}$ (b) Ground truth at time in-

Fig. 14. Ground truth and reconstructions at $t_{30}$ and at $t_{120}$ with 40 iterations for the different methods.

of alternative methods in Table II. The ground truth variable region, the final variable region estimate and the misclassified pixels are displayed in Fig. 13. A visual comparison of the different reconstructions can be done in Fig. 14. 
Visual as well as numerical results indicate the advantage of the region inconsistency minimization algorithm. The SIRT reconstruction in Fig. 14(c) and (d) is exactly the same for every time point, which makes it useless for studying structural changes within the scanned object. The conventional method's reconstruction of Fig. 14(e) and (f) is able to image changes through time, but suffers from severe streak artifacts because it is based on limited projection data per time point reconstruction. In contrast, the rSIRT reconstruction of Fig. 14(g) and (h) is able to simultaneously capture the structural changes and maintain image quality, because it combines projection data over different time points within the stationary region. This improves image quality in the stationary region and in the variable region, since the update step in rSIRT is based on difference between the simulated projections of the current reconstruction and the measured projection data.

\section{CONCLUSion}

In general, reconstructing structurally or discretely timevarying objects based on tomographic data is a difficult problem. Popular methods either reconstruct the object independently at different time points using a subset of all projection data or assume the changes to be continuous. The first method suffers from artifacts introduced by the lack of projection data per reconstruction, and the latter cannot be applied to structurally or discretely time-varying objects, as these changes are no longer representable by a continuous deformation model.

In this paper, we have presented the novel rSIRT algorithm. It can be used for the reconstruction of time-varying structurally changing objects when there exist regions within the object that remain stationary through time. There are two intuitive aspects of the rSIRT algorithm that illustrate its ability to create accurate reconstructions. On the one hand, rSIRT guarantees accurate image quality in the stationary region, since the iterative update step for this region is based on all available projection data, in contrast to the conventional method, where the stationary (and time-varying) region is reconstructed based upon information available in a subset of all projection data. On the other hand, as image quality in the stationary region improves, this is propagated to the variable region to some extent, since the update step for the variable region is computed using the projection difference of the previous estimate, which is based on the projection of stationary and variable region. Naturally, the final reconstruction quality is influenced by the amount of projection data used for generating the iterative update step in the time-varying region, which is encoded by the window size in this paper. However, for any fixed window size rSIRT improves the reconstruction quality, thereby allowing shorter acquisition times per time window. Motivated by its ability to reconstruct a wide variety of objects, SIRT was the algorithm of choice for the generation of the iterative update steps in the time-varying and stationary region within the rSIRT algorithm. However, any other iterative algorithm could be employed to generate these update steps. In more specific scanning setups, the choice of reconstruction algorithm should be tailored to the specific properties of the object under investigation, e.g. sparsity.

As it is time consuming and not always trivial to manually indicate the time-varying regions, we have developed the region inconsistency minimization procedure, an optimization algorithm that automatically estimates the time-varying regions. The method minimizes a region inconsistency measure, where the difference between simulated and measured projection data is weighted according to a factor that essentially encodes the ray intersection length and the quality of the simulated projection based on the proportion of the ray that passed through the stationary region (see Appendix B). Consequently, the region inconsistency minimization algorithm is most suited for objects where no extreme changes in reconstruction quality through the stationary region are present. However, most objects do not exhibit this kind of behavior, which makes the region inconsistency minimization method widely applicable.

Experiments with simulated and $\mu \mathrm{CT}$ data illustrate the ability of the region inconsistency minimization algorithm to find a good approximation of the time-varying regions. Also, the rSIRT algorithm was shown to have a clear advantage over popular methods when constructing structurally changing objects.

\section{APPENDIX A}

A brief description of the parametric B-spline closed curve model [30], [32] is given, which is used throughout this paper for describing the variable region within the scanned object. To define the normalized B-spline closed curve of degree $k$ with $n$ control points, we first introduce $n+2 k+1$ knot-points $t_{-k}<$ $t_{-k+1}<\cdots<t_{n+k} \in \mathbb{R}$. The following recursion relations are used to define the normalized B-spline basis functions of degree $k$ for $i=-k,-k+1, \ldots, n-1$ :

$$
\begin{aligned}
N_{i, k+1}(t)= & \frac{t-t_{i}}{t_{i+k}-t_{i}} N_{i, k}(t) \\
& +\frac{t_{i+k+1}-t}{t_{i+k+1}-t_{i+1}} N_{i+1, k}(t) \\
N_{i, 1}(t)= & \begin{cases}1 & \text { if } t_{i} \leq t<t_{i+1} \\
0 & \text { otherwise }\end{cases}
\end{aligned}
$$

The normalized B-spline $N_{i, k+1}$ can be used to define a closed curve $\boldsymbol{c}(t)$ for $t \in\left[t_{0}, t_{n}\right]$ :

$$
\boldsymbol{c}(t)=\sum_{i=-k}^{n-1} \boldsymbol{c}_{i} N_{i, k+1}(t),
$$

with control points $\boldsymbol{c}_{i} \in \mathbb{R}^{2}$ for $i=-k, \ldots, n-1$ and $\boldsymbol{c}_{i}=\boldsymbol{c}_{i-n}$ for $i=n-k, \ldots, n-1$. Once the knot-points are fixed, the region within the closed curve $c$ is completely described by the coordinates of the control points $\boldsymbol{c}_{0}, \ldots, \boldsymbol{c}_{n-1}$.

\section{APPENDIX B}

In this appendix, a more detailed description of the normalized adjusted inverse row sum matrix $\overline{\boldsymbol{R}}_{\alpha}^{\lambda}$ is given. Remember that $\overline{\boldsymbol{R}}_{\alpha}^{\lambda}$ is calculated as the normalized version of Eq. (10), 
where the normalization refers to the fact that the diagonal elements of $\boldsymbol{R}_{\alpha}^{\lambda}$ are multiplied with a suitable factor in order to have the same mean as the diagonal elements of the standard inverse row sum matrix $\boldsymbol{R}$.

Unlike $\boldsymbol{R}$, which contains weights that encode the ray intersection lengths with the reconstruction domain, $\overline{\boldsymbol{R}}_{\alpha}^{\lambda}$ additionally encodes how much of the ray interacted with the variable region. It can be understood in light of the region inconsistency measure $r I_{\lambda, 0,0}(\boldsymbol{\alpha})=\left\|\tilde{\boldsymbol{W}} \boldsymbol{S}_{K}^{\boldsymbol{\alpha}} \boldsymbol{p}-\boldsymbol{p}\right\|_{\overline{\boldsymbol{R}}_{\alpha}^{\lambda}}^{2}$ :

- If a large part of the ray passes through the variable region, its corresponding value in $\overline{\boldsymbol{R}}_{\boldsymbol{\alpha}}^{\lambda}$ will be rather small, indicating that the difference between the simulated and the measured detector value in this detector pixel is less important than others.

- If a ray passes mostly through the stationary region, the corresponding value in $\overline{\boldsymbol{R}}_{\boldsymbol{\alpha}}^{\lambda}$ will be rather large. This means that the simulated projection value should have a strong resemblance to the measured detector value.

Intuitively, rSIRT reconstructs the stationary region using all projection data, hence this part of the reconstruction should be more accurate and the calculated projection values corresponding to this region should have a good match with the measured projection data, which is encoded by the relatively larger weights in $\overline{\boldsymbol{R}}_{\alpha}^{\lambda}$. On the other hand, the variable region is reconstructed using less projection data, and is therefore expected to be of lower quality in comparison to the stationary region. This is encoded in the fact that corresponding projection values receive a smaller weight. Without this adjusted weighting, optimization of $r I$ would always result in a variable region covering the entire reconstruction domain. This can be understood as follows: The stationary regions are used to generate projections that must match up with all projection data, which limits the set of possible solutions for the pixels belonging to the stationary regions. On the other hand, the variable regions are used to simulate projections that must match up with only a subset of all projection data, which results in a larger set of possible solutions for the pixels in the variable region. As the size of the variable region increases, the set of possible reconstructions matching the projection data grows, which would result in a smaller value for the projection distance. This property was experimentally confirmed by the following simulation experiment. Consider the modified Shepp-Logan phantom of Fig. 5(e)-(h). A version of this phantom on a $500 \times 500$ pixel grid was used to generate projections over 300 time points. This projection data was used to calculate a rSIRT reconstruction with window size $n_{w}=30$ on a $100 \times 100$ grid. Subsequently $r I_{\lambda, 0,0}(\boldsymbol{\alpha})$ was calculated for different values of $\lambda$ and for different variable region's sizes, resulting in Fig. 15. Note that $\lambda=1$ corresponds to a weighting with the standard inverse row sum matrix $\boldsymbol{R}$.

\section{APPENDIX C}

This appendix provides more details about the linear operator $\boldsymbol{S}_{K}$ that represents the rSIRT algorithm. More precisely, if $\tilde{\boldsymbol{x}}^{(0)}=\mathbf{0}$, the rSIRT reconstruction after $K$ iterations depends linearly on the projection data, i.e., $\tilde{\boldsymbol{x}}^{(K)}=\boldsymbol{S}_{K} \boldsymbol{p}$.

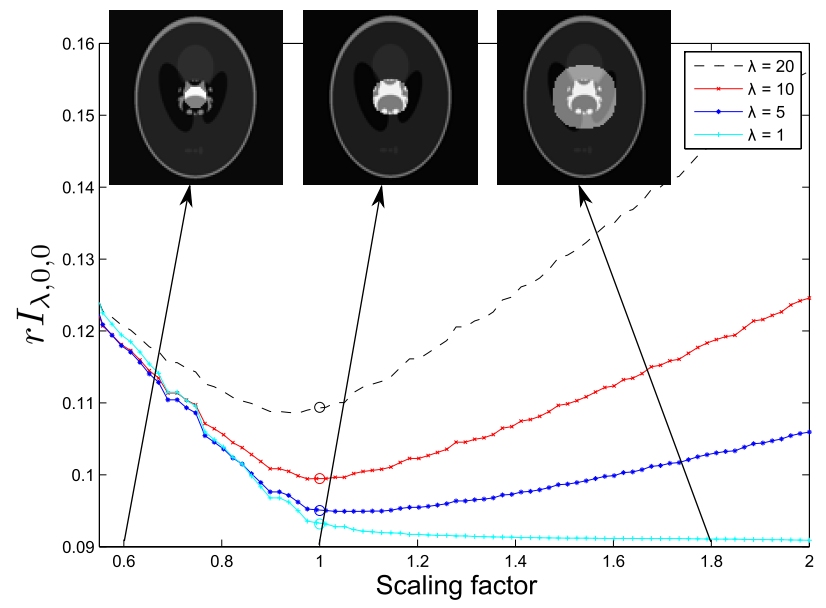

Fig. 15. The region inconsistency measure $r I_{\lambda, 0,0}(\boldsymbol{\alpha})$ for different region sizes and for $\lambda=1,5,10,20$, illustrated on the phantom of Fig. 5(e)-(h). A scaling factor 1 corresponds to the ground truth region. To illustrate the size of the variable regions for different scaling factors, we have superimposed the region for a scaling factor of $0.6,1$ and 1.8 on the phantom.

First, for $l=1, \ldots, n_{\theta}$, define $\boldsymbol{K}_{l}$ as the $N \times n_{\theta} N$ matrix that selects the reconstruction $\boldsymbol{x}_{l}$ at time index $l$ from the full reconstruction vector $\tilde{\boldsymbol{x}}$, i.e., $\boldsymbol{K}_{l} \tilde{\boldsymbol{x}}=\boldsymbol{x}_{l}$. Also define $\boldsymbol{L}_{l}$ as the $n_{w} d \times n_{\theta} d$ matrix that selects the projection data $\tilde{p}_{l}$ corresponding to the $l$ th window from the full projection data $\boldsymbol{p}$, i.e., $\boldsymbol{L}_{l} \boldsymbol{p}=\tilde{\boldsymbol{p}}_{l}$. With these definitions, Eq. (7) can be rewritten as

$$
\boldsymbol{x}_{l}^{(k+1)}=\boldsymbol{M}_{l, 1} \tilde{\boldsymbol{x}}^{(k)}+\boldsymbol{M}_{l, 2} \boldsymbol{p},
$$

with

$$
\boldsymbol{M}_{l, 1}=\boldsymbol{K}_{l}-\boldsymbol{I}_{S} \boldsymbol{C} \boldsymbol{W}^{T} \boldsymbol{R} \tilde{\boldsymbol{W}}-\boldsymbol{I}_{V} \tilde{\boldsymbol{C}}_{l} \tilde{\boldsymbol{W}}_{l}^{T} \tilde{\boldsymbol{R}}_{l} \tilde{\boldsymbol{W}}_{l} \boldsymbol{K}_{l}
$$

and

$$
\boldsymbol{M}_{l, 2}=\boldsymbol{I}_{S} \boldsymbol{C} \boldsymbol{W}^{T} \boldsymbol{R}+\boldsymbol{I}_{V} \tilde{\boldsymbol{C}}_{l} \tilde{\boldsymbol{W}}_{l}^{T} \tilde{\boldsymbol{R}}_{l} \boldsymbol{L}_{l}
$$

Set

$$
\boldsymbol{M}=\left[\begin{array}{cc}
\boldsymbol{M}_{1,1} & \boldsymbol{M}_{1,2} \\
\vdots & \vdots \\
\boldsymbol{M}_{n_{\theta}, 1} & \boldsymbol{M}_{n_{\theta}, 2} \\
\emptyset & \mathbb{1}_{n_{\theta} d}
\end{array}\right],
$$

where $\mathbb{1}_{n}$ represents the identity matrix of size $n \in \mathbb{N} \backslash\{0\}$. It can be easily verified that for

$$
\boldsymbol{S}_{K}=\left[\begin{array}{ll}
\mathbb{1}_{n_{\theta} N} & \emptyset
\end{array}\right] \boldsymbol{M}^{K}\left[\begin{array}{c}
\emptyset \\
\mathbb{1}_{n_{\theta} d}
\end{array}\right],
$$

we have $\tilde{\boldsymbol{x}}^{(K)}=\boldsymbol{S}_{K} \boldsymbol{p}$.

\section{ACKNOWLEDGMENT}

The authors are grateful to Bruker-microCT (Kartuizersweg 3B, 2550 Kontich, Belgium) for the use of their SkyScan $1172 \mu \mathrm{CT}$ scanner, and to E. Van de Casteele (iMinds-Vision Lab, University of Antwerp, Wilrijk, Belgium) for assisting with the experiments performed on this scanner. The authors wish to acknowledge W. J. Palenstijn, also from the Vision Lab, for his valuable comments and several useful discussions. 


\section{REFERENCES}

[1] R. Mooser, F. Forsberg, E. Hack, G. Székely, and U. Sennhauser, "Estimation of affine transformations directly from tomographic projections in two and three dimensions," Mach. Vis. Appl., vol. 24, no. 2, pp. 419-434, Feb. 2013.

[2] G. Van Eyndhoven, J. Sijbers, and K. J. Batenburg, "Combined motion estimation and reconstruction in tomography," in Proc. ECCV Workshops Demonstrations, Oct. 2012, pp. 12-21.

[3] T. Li, E. Schreibmann, Y. Yang, and L. Xing, "Motion correction for improved target localization with on-board cone-beam computed tomography," Phys. Med. Biol., vol. 51, no. 2, pp. 253-67, Jan. 2006.

[4] Q. Zhang, A. Pevsner, A. Hertanto, Y.-C. Hu, K. E. Rosenzweig, C. C. Ling, et al., "A patient-specific respiratory model of anatomical motion for radiation treatment planning," Med. Phys., vol. 34, no. 12, pp. 4772-4781, Dec. 2007.

[5] J. Hinkle, M. Szegedi, B. Wang, B. Salter, and S. Joshi, "4D CT image reconstruction with diffeomorphic motion model," Med. Image Anal., vol. 16, no. 6, pp. 1307-16, Aug. 2012.

[6] R. Li, X. Jia, J. H. Lewis, X. Gu, M. Folkerts, C. Men, et al., "Real-time volumetric image reconstruction and 3D tumor localization based on a single X-ray projection image for lung cancer radiotherapy," Med. Phys., vol. 37, no. 6, pp. 2822-2826, Jun. 2010.

[7] A. Docef and M. J. Murphy, "Reconstruction of 4D deformed CT for moving anatomy," Int. J. Comput. Assist. Radiol. Surgery, vol. 3, no. 6, pp. 591-598, Dec. 2008.

[8] J. Ehrhardt, R. Werner, A. Schmidt-Richberg, and H. Handels, "Statistical modeling of $4 \mathrm{D}$ respiratory lung motion using diffeomorphic image registration," IEEE Trans. Med. Imaging, vol. 30, no. 2, pp. 251-65, Feb. 2011.

[9] S. S. Vedam, P. J. Keall, V. R. Kini, H. Mostafavi, H. P. Shukla, and R. Mohan, "Acquiring a four-dimensional computed tomography dataset using an external respiratory signal," Phys. Med. Biol., vol. 48, no. 1, pp. 45-62, Jan. 2003.

[10] J. Williams, K. Yazzie, E. Padilla, N. Chawla, X. Xiao, and F. De Carlo, "Understanding fatigue crack growth in aluminum alloys by in situ X-ray synchrotron tomography," Int. J. Fatigue, vol. 57, pp. 79-85, Dec. 2013.

[11] I. Riedel, E. Andò, S. Salager, P. Bésuelle, and G. Viggiani, "Water retention behaviour explored by X-ray CT analysis," in Unsaturated Soils: Research and Applications. New York, NY, USA: Springer-Verlag, 2012, pp. 81-88.

[12] P. Gregory, D. J. Hutchison, D. B. Read, P. M. Jenneson, W. B. Gilboy, and E. J. Morton, "Non-invasive imaging of roots with high resolution X-ray micro-tomography," Plant Soil, vol. 255, no. 1, pp. 351-359, Aug. 2003.

[13] H. Gao, J.-F. Cai, Z. Shen, and H. Zhao, "Robust principal component analysis-based four-dimensional computed tomography," Phys. Med. Biol., vol. 56, no. 11, pp. 3181-98, Jun. 2011.

[14] J. Lee, J. W. Stayman, Y. Otake, S. Schafer, W. Zbijewski, A. J. Khanna, et al., "Volume-of-change cone-beam CT for image-guided surgery," Phys. Med. Biol., vol. 57, no. 15, pp. 4969-4989, Aug. 2012.

[15] R. Henderson, "The potential and limitations of neutrons, electrons and X-rays for atomic resolution microscopy of unstained biological molecules," Quart. Rev. Biophys., vol. 28, no. 2, pp. 171-193, May 1995.

[16] O. Ludwig, M. Dimichiel, and L. Salvo, "In-situ three-dimensional microstructural investigation of solidification of an $\mathrm{Al}-\mathrm{Cu}$ alloy by ultrafast X-ray microtomography," Metall. Mater. Trans. A, vol. 36, no. 6, pp. 1515-1523, Jun. 2005.

[17] P. Bésuelle, G. Viggiani, N. Lenoir, J. Desrues, and M. Bornert, "X-ray micro CT for studying strain localization in clay rocks under triaxial compression," in Proc. 2nd Int. Workshop X-Ray CT Geomater, 2006, pp. $35-52$

[18] C. Kondo, S. Mori, M. Endo, K. Kusakabe, N. Suzuki, A. Hattori, et al., "Real-time volumetric imaging of human heart without electrocardiographic gating by 256-detector row computed tomography," J. Comput. Assist. Tomogr., vol. 29, no. 5, pp. 694-698, Oct. 2005.

[19] G. Van Eyndhoven, J. Sijbers, and K. J. Batenburg, "An algebraic reconstruction technique for the study of local structural changes during CT," in Proc. 1st ICTMS, 2013, pp. 137-140.

[20] A. C. Kak and M. Slaney, Principles of Computerized Tomographic Imaging (Society of Industrial and Applied Mathematics). New York, NY, USA: Springer-Verlag, 2001.

[21] T. M. Buzug, Computed Tomography: From Photon Statistics to Modern Cone-Beam CT. New York, NY, USA: Springer-Verlag, 2008.

[22] P. M. Joseph, "An improved algorithm for reprojecting rays through pixel images," IEEE Trans. Med. Imaging, vol. 1, no. 3, pp. 192-196, Jan. 1982.
[23] B. De Man and S. Basu, "Distance-driven projection and backprojection in three dimensions," Phys. Med. Biol., vol. 49, no. 11, pp. 2463-2475, Jun. 2004.

[24] Y. Long, J. A. Fessler, and J. M. Balter, "3D forward and back-projection for X-ray CT using separable footprints," IEEE Trans. Med. Imaging, vol. 29 , no. 11 , pp. $1839-50$, Nov. 2010.

[25] J. Gregor and T. Benson, "Computational analysis and improvement of SIRT," IEEE Trans. Med. Imaging, vol. 27, no. 7, pp. 918-24, Jan. 2008

[26] K. J. Batenburg and L. Plantagie, "Fast approximation of algebraic reconstruction methods for tomography," IEEE Trans. Image Process., vol. 21, no. 8, pp. 3648-3658, Aug. 2012.

[27] W. J. Palenstijn, K. J. Batenburg, and J. Sijbers, "Performance improvements for iterative electron tomography reconstruction using graphics processing units (GPUs)," J. Struct. Biol., vol. 176, no. 2, pp. 250-253, Nov. 2011.

[28] R. Rangayyan, A. P. Dhawan, and R. Gordon, "Algorithms for limitedview computed tomography: An annotated bibliography and a challenge," Appl. Opt., vol. 24, no. 23, pp. 4000-4012, Dec. 1985.

[29] J. Moré, The Levenberg-Marquardt Algorithm: Implementation and Theory (Lecture Notes in Mathematics), vol. 630. Berlin/Heidelberg, Germany: Springer-Verlag, 1978, ch. 10, pp. 105-116.

[30] W. Boehm, "Inserting new knots into B-spline curves," Comput., Aided Des., vol. 12, no. 4, pp. 199-201, Jul. 1980.

[31] N. Otsu, "A threshold selection method from gray-level histograms," IEEE Trans. Syst., Man, Cybern., vol. 9, no. 1, pp. 62-66, Jan. 1979.

[32] C. D. Boor, "On calculating with B-splines," J. Approx. Theory, vol. 62, no. 1 , pp. 50-62, 1972

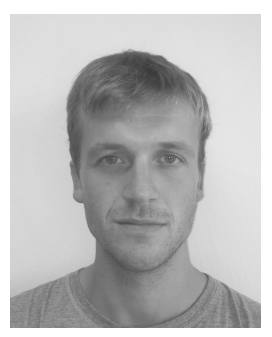

Geert Van Eyndhoven received the bachelor's degree in mathematics and later the M.Sc. degree in mathematical engineering from Katholieke Universiteit Leuven in 2011. After spending half a year in the industry, he became a Ph.D. student at the Vision Lab, Department of Physics, University of Antwerp, Belgium. His doctoral research focusses on iterative reconstruction algorithms in computed tomography.

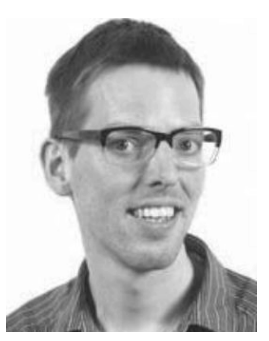

Kees Joost Batenburg received the M.Sc. degree in mathematics and the M.Sc. degree in computer science from the University of Leiden, Leiden, The Netherlands, in 2002 and 2003, respectively, and the $\mathrm{Ph} . \mathrm{D}$. degree in mathematics in 2006. He is currently a Senior Researcher with Centrum Wiskunde en Informatica, Amsterdam, where he performs research in the field of computational imaging. $\mathrm{He}$ has two part-time professorships at the University of Antwerp, Belgium, and the University of Leiden, The Netherlands.

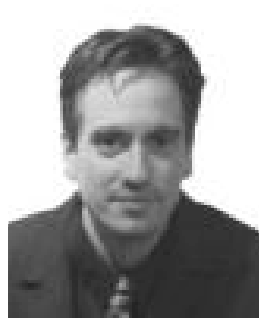

Jan Sijbers received the M.Sc. degree in physics and the Ph.D. degree in sciences/physics from the University of Antwerp, Belgium, in 1993 and 1998, respectively. He was a Post-Doctoral Fellow of the Fund for Scientific Research jointly at the University of Antwerp and the Delft University of Technology, The Netherlands, from 2002 to 2008. Since 2010, he has been a Senior Lecturer with the University of Antwerp. His main interest is in biomedical image reconstruction, processing and analysis with focus on diffusion MRI processing and iterative reconstruction for computed tomography. 